\title{
MiR-26b-5p-Modified hUB-MSCs Derived Exosomes Attenuate Early Brain Injury During Subarachnoid Hemorrhage via MAT2A-Mediated the p38 MAPK/STAT3 Signaling Pathway
}

\section{Zunwei Liu ( $\nabla$ zunweil@21cn.com )}

Nephropathy Hospital,the First Affiliated Hospital of Xi'an Jiaotong University https://orcid.org/00000002-1651-9988

\section{Bo Wang}

The First Affiliated Hospital of Xi'an Jiaotong University

\section{Qihang Guo}

Nephropathy Hospital,the First Affiliated Hospital of Xi'an Jiaotong University

\section{Research Article}

Keywords: SAH, hUC-MSCs, exosomes, miR-26b-5p, MAT2A, the p38 MAPK/STAT3 signaling pathway

Posted Date: May 28th, 2021

DOl: https://doi.org/10.21203/rs.3.rs-446068/v1

License: (c) (i) This work is licensed under a Creative Commons Attribution 4.0 International License.

Read Full License 


\section{Abstract}

Early brain injury (EBI) is a major cause of adverse outcomes following subarachnoid hemorrhage (SAH). There is evidence that mesenchymal stem cells (MSCs) - derived exosomes are involved in the repair of $\mathrm{SAH}$. Exosomes were extracted from human umbilical cord mesenchymal stem cells (hubMSCs) and identified. OxyHb treated PC12 cells were transfected with exosomes alone or together with miR-26b-5p inhibitor. Hub-MSCs derived exosomes promote cell proliferation, inhibit apoptosis and reduce inflammatory mediator expression. Transfection of miR-26b-5p inhibitor abolished the promoting effect of exosomes on the proliferation of PC12 cells, as well as the inhibitory effect on cell apoptosis. In addition, methionine adenosyltransferase II alpha (MAT2A) was one target gene of miR-26b-5p. OxyHb treated PC12 cells were transfected with exosomes alone or together with pcDNA-MAT2A and observed that the promoting effect of exosomes on PC12 cell proliferation was abolished by pcDNA-MAT2A, which was the same as the effect of miR-26b-5p inhibitor. OxyHb treated PC12 cells incubated with exosomes were transfected with miR-26b-5p inhibitor alone or together with si-MAT2A, respectively, and it was observed that exosomes decreased the phosphorylation levels of p38 MAPK and STAT3 proteins, inhibited cell apoptosis and inflammatory mediator expression, and miR-26b-5p inhibitor abrogated the effects of exosomes, while transfection of si-MAT2A reversed the effects of miR-26b-5p inhibitor. Moreover, injection of miR-26b-5p inhibitor resulted in increased MAT2A and pathway protein expression, increased inflammatory mediators, and aggravated neurological symptoms in the brain tissues of SAH rats.

\section{Introduction}

Subarachnoid hemorrhage (SAH) refers to a clinical syndrome caused by rupture of blood vessels at the base or surface of the brain and homeopathic flow of blood into the subarachnoid space, accounting for about $10 \%$ of acute stroke[14]. The pathophysiological mechanisms of SAH are mainly early brain injury (EBI) and delayed brain injury (DBI) after subarachnoid hemorrhage. In the past, cerebral vasospasm was considered to be the main cause of neurological injury and cognitive impairment after SAH, but increasing evidence suggests that $\mathrm{EBI}$ is the more important factor affecting the poor prognosis of $\mathrm{SAH}$ patients[7, 3, 25], so in-depth study of EBI will be helpful to propose treatment methods to improve the prognosis of SAH patients.

Stem cells are known as seed cells for repair and reconstruction of damaged tissues. Among them, mesenchymal stem cells (MSCs) are adult stem cells derived from the early stage of mesoderm development with the potential of self-repair and multi-differentiation[4, 26]. Studies have found that intraventricular transplantation of human umbilical cord mesenchymal stem cells (hUC-MSCs) significantly alleviates SAH-induced chronic hydrocephalus, inflammatory cytokine secretion and behavioral disorders, and knockdown of transforming growth factor-beta 1 expression in hUC-MSCs enhances these effects[2]. In addition, one study found that SAH rats transplanted with bone marrow mesenchymal stem cells (BMSCs) showed reduced neurological dysfunction, decreased expression of pro-inflammatory factors including interleukin (IL)-1 $\beta$, IL-6 and TNF- $\alpha$ in the left hemisphere, and 
increased secretion of anti-inflammatory factor IL-10[11]. When BMSCs were co-incubated with oxyhemoglobin-activated BV-2 microglia, it was observed that oxyHb-induced BV2 activation and polarization were inhibited, and the level of inflammatory genes was strongly downregulated[27].

Withing the deepening of research, exosomes derived from MSCs have attracted extensive attention and are considered as potential drug carriers, which are expected to replace MSCs transplantation. Exosomes are microvesicle-like structures produced and secreted by cells, containing a variety of proteins, mRNAs, miRNAs and other bioactive substances, which not only participate in cell transport and cell communication, but also participate in cell composition as components of organelles $[5,15,9]$. Increased evidence suggests that MSCs-derived exosomes are involved in the repair of brain injury. One study showed that traumatic brain injury animals treated with MSCs-derived exosomes at the same time exhibited shorter neurological recovery cycles and less brain damage than saline alone[20]. After systemic administration of exosomes derived from MSCs to Wistar rats with experimental traumatic brain injury, it was observed that the sensorimotor function of rats recovered, the number of neonatal endothelial cells in the lesion boundary zone increased, the number of neonatal mature neurons in the dentate gyrus increased, and the neuroinflammation disappeared[29]. Other studies showed that BMSCsexosomes significantly improved neurological function, decreased brain water content, maintained the integrity of the blood-brain barrier, and attenuated neuroinflammation in SAH rats, which was mediated by the inhibition of HMGB1-TLR4 pathway activity by miR-129-5p in exosomes[23].

Recently, there is evidence that exosomes miR-26b-5p derived from hUC-MSCs can inhibit M1 polarization of microglia by inactivating toll-like receptor pathway by targeting $\mathrm{CH} 25 \mathrm{H}$, thereby alleviating neurological injury after cerebral ischemia/reperfusion[8]. However, the regulatory role of exosome miR-26b-5p derived from hUC-MSCs in early brain injury is still unclear, so this study explored the pathological mechanism of its involvement in $\mathrm{SAH}$, aiming to provide a new idea for the treatment of SAH.

\section{Materials And Methods \\ 2.1 Animals}

The healthy adult SD rats used in this study were provided by the Laboratory Animal Center of China Medical University, weighing 300-320 g. All experimental animals were raised by professionals under conditions of $12 \mathrm{~h} / 12 \mathrm{~h}$ light and dark cycles per day, and constant indoor temperature and humidity with free access to food and water. Establishment of SAH animal model by classical occipital cistern secondary blood injection method[13]. Thirty SD rats were randomly divided into five groups, six in each group, namely: Sham (injection of saline into the cisterna magna), SAH (tail vein injection, $100 \mu \mathrm{g} / \mathrm{mL}$ PBS), SAH + exosomes (tail vein injection, $100 \mu \mathrm{g} / \mathrm{mL}$ ), SAH + exosomes + NC inhibitor, SAH + exosomes + miR-26b-5p inhibitor. This study was approved and supervised by the Committee of XX Laboratory Animal Center, and the experimental process conformed to the principles of the use and protection of experimental animals.

\subsection{Cell culture and Transfection}


HUC-MSCs and PC12 cells were purchased from the American Typical Culture Collection (ATCC, Manassas, VA, USA). Cells were incubated in containing $10 \%$ fetal bovine serum, $100 \mathrm{U} / \mathrm{mL}$ penicillin and $100 \mu \mathrm{g} / \mathrm{mL}$ streptomycin (Sigma,St. Louis, MO, USA) in RPMI 1640 medium (Gibco, Rockville, MD). When the cells grew vigorously and the fusion degree was about $80 \%$, they were digested with $1 \mathrm{~mL}$ of $0.25 \%$ trypsin. When most of the cells were observed to be shrunken and rounded and floating on the liquid surface in a flowing state, $3 \mathrm{~mL}$ DMEM was added to terminate digestion and passaged at a ratio of 1:2. The miR-26b-5p inhibitor and negative control inhibitor were purchased from Santa Cruz Biotechnology (Santa Cruz, CA, USA). Transfection was performed using Lipofectamine 2000 transfection reagent (Invitrogen, Carlsbad, CA, USA) according to the manufacturer's instructions. The pcDNA3.1 and pcDNAMAT2A were purchased from RiboBio Co., Ltd (Guangzhou, China) and transfected into cells according to the instructions.

\subsection{Isolation and extraction of exosomes}

The exosomes derived from hUC-MSCs were extracted using the exosomes extraction kits, and the specific operation was as follows. The P2 generation hUC-MSCs were incubated in DMEM medium without exosomes for $24 \mathrm{~h}$, and $20 \mathrm{~mL}$ cell culture supernatant was collected, centrifuged at $3,000 \times \mathrm{g}$ for $30 \mathrm{~min}$, and the precipitation was discarded. The supernatant was taken and the extraction reagent was added at a ratio of $5: 1$. The supernatant was placed at $4^{\circ} \mathrm{C}$ for $12 \mathrm{~h}$, and then centrifuged at $100,000 \times \mathrm{g}$ for 60 min to obtain exosomes precipitation. The PBS was resuspended and repeatedly blown with a pipette gun until the precipitate was dissolved. The concentration was adjusted to be about 50 and 100 $\mu \mathrm{g} / \mathrm{mL}$. The samples were packaged and stored at $-80^{\circ} \mathrm{C}$ for subsequent experiments.

\subsection{RT-qPCR}

Total RNA was isolated from PC12 cell by using the TRIzol (Invitrogen, Carlsbad, CA, USA). Then the first strand of cDNA was synthesized using MMLV Reverse Transcriptase kit (TaKaRa, Da Lian, China). Realtime PCR was conducted by using SYBR Premix Ex TaqTM Kit (Applied Biosystems, Foster City, CA, USA). The reaction was run in ABI7500 Real-time PCR system (Applied Biosystems, Carlsbad, CA). U6 were used as an endogenous control. Briefly, $2 \mu \mathrm{L}$ of CDNA was added to $10 \mu \mathrm{L}$ of the $2 \times$ SYBR green PCR master

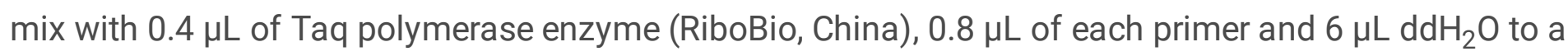
final volume of $20 \mu \mathrm{L}$. The RT-qPCR cycling conditions consisted of: $95^{\circ} \mathrm{C}$ for $3 \mathrm{~min}$; then 35 cycle amplification for $20 \mathrm{~s}$ at $95^{\circ} \mathrm{C}, 30 \mathrm{~s}$ at $55^{\circ} \mathrm{C}, 15 \mathrm{~s}$ at $72^{\circ} \mathrm{C}$; followed by $1 \mathrm{~min}$ at $72^{\circ} \mathrm{C}$. The primers used in this study were synthesized by Sangon Biotech (Shanghai, China). The $2^{-\Delta \Delta C t}$ method was used for quantitative analysis.

\subsection{MTT assay}

When the density of cells reached $80 \%$, cells were digested with $0.25 \%$ trypsin, and single cell suspension was made with DMEM medium containing $10 \%$ serum. $200 \mu \mathrm{L}$ of culture medium containing $2 \times 10^{3}$ cells were added into each well of 96 -well plate, and incubated in a $5 \% \mathrm{CO}_{2}$ incubator at $37^{\circ} \mathrm{C}$ for $72 \mathrm{~h}$.

According to the time point, add $20 \mu \mathrm{L}$ of $5 \mathrm{mg} / \mathrm{mL}$ MTT solution into each hole, continue to culture for 4 $\mathrm{h}$, then stop the culture, discard the culture supernatant; add $150 \mu \mathrm{L}$ DMSO into each hole and shake for 
10 min to dissolve the crystals fully, and measure the absorbance value of each hole at $490 \mathrm{~nm}$ wavelength. Each experimental procedure was processed at least three times.

\subsection{Flow cytometry}

Cells were digested with $0.25 \%$ trypsin, rinsed with PBS three times, and all collected cells were transferred to a $15 \mathrm{~mL}$ centrifuge tube, centrifuged at 1,500 rpm for $5 \mathrm{~min}$, and the supernatant was discarded. The cells were washed with PBS, and the supernatant was discarded by centrifugation and repeated twice. $400 \mu \mathrm{L}$ PBS was added to resuspend the cells, while $2 \mathrm{~mL}$ of pre-cooled ethanol was added, and the cells were rested at $-20^{\circ} \mathrm{C}$ for $30 \mathrm{~min}$. Next, the cells were centrifuged at $1,500 \mathrm{rpm}$ for 5 min, resuspended with $1000 \mu \mathrm{L}$ PBS, separated at 1,500 rpm for 5 min, and incubated for 30 min in the dark after staining, then apoptosis was detected by flow cytometry.

\subsection{Luciferase Reporter Gene Assay}

StarBase (http://starbase.sysu.edu.cn/) was used to predict potential target genes of miR-26b-5p, and MAT2A was screened as the research object. The wild type and mutant 3'-UTR sequences of MAT2A were cloned into the PGL3 vector (Promega, USA) fused luciferase gene sequence, respectively. HEK293 cells were seeded in 24-well plates, and when growing to approximately 70\% confluence, co-transfected with luciferase plasmids and miR-26b-5p mimic or NC mimic using Lipofectamine 2000. After $48 \mathrm{~h}$ of transfection, the luciferase reporter activity was measured by Dual-Luciferase Reporter Assay System (Promega, Madison, WI) under the manufacturer's instructions.

\subsection{Western blotting}

Total protein was extracted from cells or brain tissues using Pro-prep TM protein Extraction Solution (Daejeon, Korea). The protein content of each sample was determined using the BCA Protein Assay Kits (Thermo Scientific). Then, equal amounts of proteins (15 $\mu \mathrm{g} / \mathrm{lane}$ ) were separated on a $12 \%$ sodium dodecyl sulfate polyacrylamide gel electrophoresis (SDS-PAGE) and transferred to polyvinylidenedifluoride (PVDF) membranes (Bio-Rad, Hercules, CA, USA). The membranes were blocked in $5 \%(\mathrm{w} / \mathrm{v})$ nonfat dry milk in TBST (Tris-buffered saline-0.1\%Tween) at $25^{\circ} \mathrm{C}$ for $3 \mathrm{~h}$ and then incubated with the following primary antibodies: rabbit polyclonal anti- $\beta$-actin antibody (1:1000, Abcam, ab8227), rabbit monoclonal anti-CD63 antibody (1:1500, Abcam, ab217345), mouse monoclonal anti-CD81 antibody (1:1200, Abcam, ab79559), rabbit monoclonal anti-TSG101 antibody (1:2500, Abcam, ab125011), rabbit monoclonal anti-Alix antibody (1:2000, Abcam, ab186492), Rabbit polyclonal antiMAT2A antibody (1:2500, Abcam, ab154343), Rabbit monoclonal anti-COX-2 antibody (1:2000, Abcam, ab15191), Rabbit monoclonal anti-MCP-1 antibody (1:1000, Abcam, ab214819), Rabbit monoclonal antiiNOS antibody (1:800, Abcam, ab178945), rabbit monoclonal anti-STAT3 antibody (1:1500, Abcam, ab68153), rabbit monoclonal anti-AKT (phospho Y705) antibody (1:5000, Abcam, ab76315). Then, the membranes were incubated with horseradish peroxidase (HRP)-conjugated goat anti-rabbit IgG (1:3000, Abcam, ab6721) or goat anti-mouse IgG (1:3000, Abcam, ab6728) for $1 \mathrm{~h}$ at room temperature. The bands were visualized by using an ECL Plus Chemiluminescence Reagent Kit (Pierce, Rockford, IL, USA) 
and were photographed by a chemiluminescence imaging system. Image $\mathrm{J}$ software was used to quantify the band densities.

\subsection{Statistical Analysis}

All statistical analyses were performed by using the SPSS software (ver.22.0; SPSS, Chicago, IL). All data were shown as mean \pm SD. Comparisons between two groups were made by the Student's $t$-test. Data between multiple groups were performed with one-way analysis of variance (ANOVA) followed by post hoc analysis with LSD test. P®0.05 was considered statistical significance.

\section{Results}

\subsection{Identification of exosomes derived from hUC-MSCs}

HUC-MSCs culture supernatant was collected, and then cell-derived exosomes were extracted with a kit and purified by ultracentrifugation. Westem Blotting was used to detect the expression of exosomes marker proteins in the precipitates isolated from hUC-MSCs. The results showed that the expression of exosomes marker proteins including CD63, CD81, Alix and TSG101 was strongly positive in the extracts isolated and purified from hUC-MSCs, which confirmed that the obtained extracts were exosomes (Fig. 1A\&1B). The conditioned medium (CM) supplemented with exosomes inhibitor GW4869 was used as the control.

\subsection{Effects of exosomes derived from hUC-MSCs on oxyHb- induced PC12 cells}

It is well known that neuronal apoptosis and inflammatory response are the main causes of early brain injury caused by $\mathrm{SAH}$. Here, we used oxyHb to induce PC12 neurons to simulate the SAH model in vitro, and further explored the effects of exosomes derived from hUC-MSCs on injured cells. We found that oxyHb induction significantly inhibited cell proliferation, promoted cell apoptosis, and increased the expression of inflammatory mediators including COX-2, MCP-1 and iNOS in cells compared with the control group. Moreover, oxyHb-induced PC12 cells were treated with exosomes at concentrations of 50 and $100 \mu \mathrm{g} / \mathrm{mL}$, respectively, and we observed enhanced cell proliferation (Fig. 2A), decreased apoptosis (Fig. 2B), and decreased expression of inflammatory mediators (Fig. 2C) in the cells. Similarly, we found that exosomes with a concentration of $100 \mu \mathrm{g} / \mathrm{mL}$ had a more significant regulatory effect on cells.

\subsection{Effects of exosomes derived from hUC-MSCs modified by miR-26b-5p on oxyHb-induced PC12 cells}

Studies have reported that exosomes miR-26b-5p derived from hUC-MSCs has an inhibitory effect on neuroinflammation, but its potential regulatory role in SAH is still worth studying. Next, we explored the effect of exosomes derived from hUC-MSCs on oxyHb-induced expression of miR-26b-5p in PC12 cells. 
We found that the expression of miR-26b-5p was significantly down-regulated in oxyHb-induced PC12 cells compared with the control group, however, the expression of miR-26b-5p was restored when oxyHbinduced PC12 cells were treated with exosomes at a concentration of $100 \mu \mathrm{g} / \mathrm{mL}$. Interestingly, transfection of the inhibitor of miR-26b-5p in injured cells incubated with exosomes, we observed that the expression of miR-26b-5p was decreased again (Fig. 3A). Furthermore, oxyHb inhibited cell proliferation, promoted apoptosis and secretion of inflammatory mediators, while exosomes treatment significantly reversed the effect of oxyHb on cells, but with the intervention of miR-26b-5p inhibitor, we found that the protective effect of exosomes on injured cells was counteracted, manifested by the weakened cell proliferation ability (Fig. 3B), increased apoptosis (Fig. 3C) and increased secretion levels of inflammatory mediators (Fig. 3D).

\subsection{MAT2A was a direct target of miR-26b-5p}

StarBase3.0 (http://starbase.sysu.edu.cn/) was used to predict potential target genes of miR-26b-5p, and MAT2A was screened as the research object. The wild type and mutant 3'-UTR sequences of MAT2A were shown in Fig. 4A. Next, the luciferase reporter gene analysis showed that miR-26b-5p mimic significantly reduced the luciferase activity of wild-type MAT2A, but had no significant effect on the luciferase activity of mutant-type MAT2A (Fig. 4B). To further verify the target relationship between miR-26b-5p and MAT2A, we bi-directionally regulated the expression of miR-26b-5p in cells. Western blotting results showed that miR-26b-5p mimic significantly downregulated the protein expression of MAT2A. Conversely, transfection of miR-26b-5p inhibitor promoted the expression of MAT2A (Fig. 4C).

\subsection{Effect of regulating MAT2A expression on oxyHb- induced PC12 cells}

Previous studies found that oxyHb-induced expression of miR-26b-5p was significantly downregulated in PC12 cells, and we confirmed that miR-26b-5p directly targeted the 3'UTR of MAT2A. Here, we detected the expression of MAT2A in PC12 cells induced by oxyHb and further explored the effect of regulating MAT2A expression on cells. As expected, oxyHb alone induced an increase in the protein expression of MAT2A in PC12 cells. In the combination treatment group, we observed that the protein expression of MAT2A was inhibited, but this inhibition was counteracted after overexpression of MAT2A (Fig. 5A). Moreover, overexpression of MAT2A in injured cells incubated with exosomes significantly inhibited cell proliferation (Fig. 5B), promoted apoptosis (Fig. 5C) and upregulated the secretion levels of inflammatory mediators including COX-2, MCP-1 and iNOS (Fig. 5A\&5D).

\subsection{MiR-26b-5p-modified exosomes inhibited p38 MAPK/STAT3 signaling pathway to alleviate oxyHb-induced cell injury}


In this study, we found that the phosphorylation levels of p38 MAPK and STAT3 proteins, apoptosis and secretion of inflammatory mediators were decreased in exosomes-treated cells compared with oxyHb alone induction (Fig. 6A). As mentioned earlier, inhibition of miR-26b-5p expression in exosomes-treated cells significantly reversed the protective effect of exosomes on oxyHb-induced cell injury. Furthermore, we simultaneously inhibited the expression of miR-26b-5p and MAT2A in cells treated with oxyHb and exosomes. The results showed that knockdown of MAT2A expression decreased the phosphorylation levels of p38 MAPK and STAT3 proteins, decreased apoptosis (Fig. 6B), and inhibited the secretion of inflammatory (Fig. 6C) mediators compared with transfection of miR-26b-5p inhibitor alone.

\subsection{MiR-26b-5p-modified exosomes attenuate early brain injury in SAH rats}

Next, we used the classical occipital cistern secondary blood injection method to establish an animal model of SAH and validate the neuroprotective effect of miR-26b-5p-modified exosomes in in vivo. Compared with sham group, the expression of miR-26b-5p was down-regulated, the expression of MAT2A protein was increased, and the phosphorylation levels of pathway-related proteins were increased in brain tissue of SAH rats. Compared with SAH group, exosomes significantly upregulated the expression of miR26b-5p (Fig. 7A), decreased the expression of MAT2A protein, and decreased the phosphorylation levels of pathway-related proteins (Fig. 7B), but the regulatory effect of exosomes was significantly reversed by miR-26b-5p inhibitor. Furthermore, $24 \mathrm{~h}$ after modeling, exosomes significantly alleviated neurological symptoms (Fig. 7C) and brain edema (Fig. 7D) in SAH rats, but transfection of miR-26b-5p inhibitor reversed the neuroprotective effect of exosomes. Similarly, we observed that miR-26b-5p inhibitor reversed the inhibitory effect of exosomes on the production of inflammatory mediators (Fig. 7E).

\section{Discussion}

Numerous studies have shown that neuroinflammation and blood-brain barrier disruption are the two main pathological mechanisms of early brain injury after $\mathrm{SAH}[21]$. After $\mathrm{SAH}$, blood rapidly enters the subarachnoid space, resulting in increased intracranial pressure, decreased cerebral blood flow, and transient cerebral ischemia. Stimulated by erythrocyte lysates and transient cerebral ischemia, intracellular inflammatory pathways are activated, and a large number of inflammatory mediators are synthesized and released, ultimately leading to brain edema[22, 10]. What's worse, neuronal cells undergo apoptosis under stress conditions, which directly affects various neural activities. After the blood-brain barrier is destroyed, the permeability increases, which promotes the infiltration of inflammatory cells, especially neutrophils, and the occurrence of brain edema, further aggravating the inflammatory response, leading to neurological deficits after $\mathrm{SAH}[24]$. Therefore, alleviating the inflammatory response after $\mathrm{SAH}$ is expected to improve the prognosis of SAH patients.

To promote functional recovery in patients with nerve injury, researchers have attempted to apply MSCs transplantation to restore normal nerve function. With the deepening of research, more evidence points out that transplanted cells promote endogenous nerve remodeling and enhance angiogenesis by 
paracrine way, and then improve the recovery of nerve function after brain injury. At present, exosomes or extracellular vesicles derived from MSCs have been widely used in animal models including traumatic brain injury, cerebral ischemia and cerebral hemorrhage.

Studies have shown that MSCs-derived exosomes significantly increase cerebrovascular density and the number of new neurons, inhibit the activation of astrocytes and microglia, and alleviate neurological symptoms in traumatic brain injury rats[28]. In a rat striatal hemorrhage model, systemic administration of MSCs-derived exosomes improved spatial memory, learning, motor and sensory memory functions in rats, possibly by promoting angiogenesis and axonal remodeling[6]. In addition, compared with PBS treatment, BMSCs-derived exosomes significantly inhibited the expression of pro-apoptotic proteins and pro-inflammatory cytokines, while down-regulating the expression of iNOS and up-regulating Arg1 expression to regulate microglia/macrophage polarization, thereby reducing neuroinflammation in mice with craniocerebral injury[12].

In this study, we observed that hUC-MSCs derived exosomes reduced oxyHb-induced apoptosis and production of inflammatory mediators. In-depth study revealed that the protective effect of exosomes on PC12 neurons was mediated by miR-26b-5p. Mechanistic studies showed that exosomes derived from hUC-MSCs modified by miR-26b-5p blocked the MAPK/STAT3 signal transduction system to promote neurological recovery by inhibiting MAT2A expression. MAT2A belongs to the adenosylmethionine transferase family, whose members, under the action of ATPase, can catalyze methionine to generate important methyl donors in organisms, and then regulate the methylation of DNA and histones[30]. Unfortunately, the study of MAT2A in cerebral hemorrhagic diseases has not been reported yet, but the related studies in other diseases can still support our conclusion. Some studies have found that MAT2A is up-regulated in TGF- $\beta 1$-induced hepatic stellate cell, induces p 65 phosphorylation, activates the NF-KB transduction system, and aggravates liver fibrosis. Inhibiting the expression of MAT2A with NPLC0393, a small molecule inhibitor of TGF- $\beta$ 1, decreased the phosphorylation level of $p 65$, but overexpressing MAT2 abolished the therapeutic effect of NPLC0393[17]. Other studies have claimed that granulocytemacrophage colony-stimulating factor signaling in astrocytes can drive MAT2A expression and proinflammatory factor transcription, thereby increasing the risk of multiple sclerosis[19].

Early brain injury caused by subarachnoid hemorrhage is mainly related to neuronal apoptosis and inflammatory response. A large number of studies have confirmed that MAPK and STAT3 signal transduction systems play important roles in inflammation, stress response, cell survival and apoptosis. The expression of MMP-9 protein and phosphorylated p38 MAPK in the sciatic nerve has been found to be positively correlated with the severity of the disease. Treatment with p38 MAPK inhibitor (SB203580) significantly reduced the levels of MMP-9 mRNA and protein in the sciatic nerve at the peak of autoimmune neuritis, and alleviated the severity of the nerve lesion[16].In the progression of intracerebral hemorrhage, Leucine-rich repeat kinase 2 has been reported to mediate inflammatory response, cognitive dysfunction, blood-brain barrier damage, and neuronal apoptosis via the p38 MAPK/Drosha pathway, thereby accelerating secondary brain injury[1]. Similarly, a large body of evidence suggests that the STAT3 pathway is involved in the development of early brain injury after SAH. Recent studies have shown 
that recombinant human erythropoietin inhibits brain cell apoptosis, neuronal necrosis, albumin exudation and brain edema in experimental SAH by downregulating the phosphorylation levels of JAK2 and STAT3 proteins in the cortex[18].

In conclusion, our findings further support the positive role of MSCs-derived exosomes in brain injury repair, which provides a new reference for improving the prognosis of SAH patients.

\section{Abbreviations}

\section{$\mathrm{SAH}$}

subarachnoid hemorrhage; EBI:early brain injury; DBI:delayed brain injury; MSCs:mesenchymal stem cells; hUC-MSCs:human umbilical cord mesenchymal stem cells; BMSCs:bone marrow mesenchymal stem cells; IL-1 $\beta$ :interleukin-1 $\beta$; IL-6:interleukin-6; IL-10:interleukin-10; TNF-a:tumor necrosis factor-a; TLR4:tolllike receptor 4; DMEM:Dulbecco's modified Eagle's medium; SDS-PAGE:sodium dodecyl sulfate polyacrylamide gel electrophoresis; PVDF:polyvinylidene difluoride; HRP:horseradish peroxidase; MAT2A:methionine adenosyltransferase II alpha; MCP-1:monocyte chemotactic protein-1

\section{Declarations}

\section{Authors' Contributions}

Zunwei Liu: Conceptualization, Supervision, Writing-original draft; Bo Wang: Methodology, Resources, Data curation; Qihang Guo: Formal analysis, Software, Writing-review \& editing.

\section{Declaration of Competing Interest}

The authors declare no competing financial interests regarding the publication of the present study.

\section{Formatting of funding sources}

This research did not receive any specific grant from funding agencies in the public, commercial, or notfor-profit sectors.

\section{Availability of data and materials}

The datasets used during the present study are available from the corresponding author upon reasonable request.

\section{Acknowledgements}

We acknowledge and appreciate our colleagues for their valuable efforts and comments on this paper.

\section{References}


1. Cao J, Zhuang Y, Zhang J, Zhang Z, Yuan S, Zhang P et al (2018) Leucine-rich repeat kinase 2 aggravates secondary brain injury induced by intracerebral hemorrhage in rats by regulating the P38 MAPK/Drosha pathway. Neurobiol Dis 119:53-64. doi:10.1016/j.nbd.2018.07.024

2. Chen H, Chen L, Xie D, Niu J (2020) Protective Effects of Transforming Growth Factor- $\beta 1$ Knockdown in Human Umbilical Cord Mesenchymal Stem Cells against Subarachnoid Hemorrhage in a Rat Model. Cerebrovasc Dis 49(1):79-87. doi:10.1159/000505311

3. Chen S, Ma Q, Krafft PR, Hu Q, Rolland W 2nd, Sherchan P et al (2013) P2X7R/cryopyrin inflammasome axis inhibition reduces neuroinflammation after SAH. Neurobiol Dis 58:296-307. doi:10.1016/j.nbd.2013.06.011

4. Faal T, Phan DTT, Davtyan H, Scarfone VM, Varady E, Blurton-Jones M et al (2019) Induction of Mesoderm and Neural Crest-Derived Pericytes from Human Pluripotent Stem Cells to Study BloodBrain Barrier Interactions. Stem Cell Reports 12(3):451-460. doi:10.1016/j.stemcr.2019.01.005

5. Fan J, Xu G, Chang Z, Zhu L, Yao J (2020) miR-210 transferred by lung cancer cell-derived exosomes may act as proangiogenic factor in cancer-associated fibroblasts by modulating JAK2/STAT3 pathway. Clin Sci (Lond) 134(7):807-825. doi:10.1042/cs20200039

6. Han Y, Seyfried D, Meng Y, Yang D, Schultz L, Chopp M et al (2018) Multipotent mesenchymal stromal cell-derived exosomes improve functional recovery after experimental intracerebral hemorrhage in the rat. J Neurosurg 131(1):290-300. doi:10.3171/2018.2.jns171475

7. Hou G, Chen H, Yin Y, Pan Y, Zhang X, Jia F (2020) MEL Ameliorates Post-SAH Cerebral Vasospasm by Affecting the Expression of eNOS and HIF1a via H19/miR-138/eNOS/NO and H19/miR675/HIF1a. Mol Ther Nucleic Acids 19:523-532. doi:10.1016/j.omtn.2019.12.002

8. Li G, Xiao L, Qin H, Zhuang Q, Zhang W, Liu L et al (2020) Exosomes-carried microRNA-26b-5p regulates microglia M1 polarization after cerebral ischemia/reperfusion. Cell Cycle 19(9):10221035. doi:10.1080/15384101.2020.1743912

9. Li J, Ding Z, Li Y, Wang W, Wang J, Yu H et al (2020) BMSCs-Derived Exosomes Ameliorate Pain Via Abrogation of Aberrant Nerve Invasion in Subchondral Bone in Lumbar Facet Joint Osteoarthritis. J Orthop Res 38(3):670-679. doi:10.1002/jor.24497

10. Liu L, Fujimoto M, Nakano F, Nishikawa H, Okada T, Kawakita F et al (2018) Deficiency of Tenascin-C Alleviates Neuronal Apoptosis and Neuroinflammation After Experimental Subarachnoid Hemorrhage in Mice. Mol Neurobiol 55(11):8346-8354. doi:10.1007/s12035-018-1006-z

11. Liu W, Li R, Yin J, Guo S, Chen Y, Fan H et al (2019) Mesenchymal stem cells alleviate the early brain injury of subarachnoid hemorrhage partly by suppression of Notch1-dependent neuroinflammation: involvement of Botch. J Neuroinflammation 16(1):8. doi:10.1186/s12974-019-1396-5

12. Ni H, Yang S, Siaw-Debrah F, Hu J, Wu K, He Z et al (2019) Exosomes Derived From Bone Mesenchymal Stem Cells Ameliorate Early Inflammatory Responses Following Traumatic Brain Injury. Front Neurosci 13:14. doi:10.3389/fnins.2019.00014

13. Pu T, Zou W, Feng W, Zhang Y, Wang L, Wang H et al (2019) Persistent Malfunction of Glymphatic and Meningeal Lymphatic Drainage in a Mouse Model of Subarachnoid Hemorrhage. Exp Neurobiol 
28(1):104-118. doi:10.5607/en.2019.28.1.104

14. Qin X, Akter F, Qin L, Xie Q, Liao X, Liu R et al (2019) MicroRNA-26b/PTEN Signaling Pathway Mediates Glycine-Induced Neuroprotection in SAH Injury. Neurochem Res 44(11):2658-2669. doi:10.1007/s11064-019-02886-2

15. Song Z, Zhang JH (2020) Recent Advances in Stem Cell Research in Subarachnoid Hemorrhage. Stem Cells Dev 29(4):178-186. doi:10.1089/scd.2019.0219

16. Sun Y, Chen H, Ma S, Liang L, Zheng Y, Guo X et al (2015) Administration of SB203580, a p38 MAPK Inhibitor, Reduced the Expression of MMP9, and Relieved Neurologic Severity in the Experimental Autoimmune Neuritis (EAN) in Rats. Neurochem Res 40(7):1410-1420. doi:10.1007/s11064-0151608-Z

17. Wang K, Fang S, Liu Q, Gao J, Wang X, Zhu H et al (2019) TGF- $\beta 1 / p 65 / M A T 2 A$ pathway regulates liver fibrogenesis via intracellular SAM. EBioMedicine 42:458-469. doi:10.1016/j.ebiom.2019.03.058

18. Wei S, Luo C, Yu S, Gao J, Liu C, Wei Z et al (2017) Erythropoietin ameliorates early brain injury after subarachnoid haemorrhage by modulating microglia polarization via the EPOR/JAK2-STAT3 pathway. Exp Cell Res 361(2):342-352. doi:10.1016/j.yexcr.2017.11.002

19. Wheeler MA, Clark IC, Tjon EC, Li Z, Zandee SEJ, Couturier CP et al (2020) MAFG-driven astrocytes promote CNS inflammation. Nature 578(7796):593-599. doi:10.1038/s41586-020-1999-0

20. Williams AM, Dennahy IS, Bhatti UF, Halaweish I, Xiong Y, Chang P et al (2019) Mesenchymal Stem Cell-Derived Exosomes Provide Neuroprotection and Improve Long-Term Neurologic Outcomes in a Swine Model of Traumatic Brain Injury and Hemorrhagic Shock. J Neurotrauma 36(1):54-60. doi:10.1089/neu.2018.5711

21. Xie Y, Peng J, Pang J, Guo K, Zhang L, Yin S et al (2020) Biglycan regulates neuroinflammation by promoting M1 microglial activation in early brain injury after experimental subarachnoid hemorrhage. J Neurochem 152(3):368-380. doi:10.1111/jnc.14926

22. Xie Z, Huang L, Enkhjargal B, Reis C, Wan W, Tang J et al (2018) Recombinant Netrin-1 binding UNC5B receptor attenuates neuroinflammation and brain injury via PPARY/NFKB signaling pathway after subarachnoid hemorrhage in rats. Brain Behav Immun 69:190-202.

doi:10.1016/j.bbi.2017.11.012

23. Xiong L, Sun L, Zhang Y, Peng J, Yan J, Liu X (2020) Exosomes from Bone Marrow Mesenchymal Stem Cells Can Alleviate Early Brain Injury After Subarachnoid Hemorrhage Through miRNA129-5pHMGB1 Pathway. Stem Cells Dev 29(4):212-221. doi:10.1089/scd.2019.0206

24. Xu H, Li J, Wang Z, Feng M, Shen Y, Cao S et al (2017) Methylene blue attenuates neuroinflammation after subarachnoid hemorrhage in rats through the Akt/GSK-3 $\beta / M E F 2 D$ signaling pathway. Brain Behav Immun 65:125-139. doi:10.1016/j.bbi.2017.04.020

25. Yin C, Huang GF, Sun XC, Guo Z, Zhang JH (2016) Tozasertib attenuates neuronal apoptosis via DLK/JIP3/MA2K7/JNK pathway in early brain injury after SAH in rats. Neuropharmacology 108:316-323. doi:10.1016/j.neuropharm.2016.04.013 
26. Zhang H, Nieves JL, Fraser ST, Isern J, Douvaras P, Papatsenko D et al (2014) Expression of podocalyxin separates the hematopoietic and vascular potentials of mouse embryonic stem cellderived mesoderm. Stem Cells 32(1):191-203. doi:10.1002/stem.1536

27. Zhang L, Guo K, Yin S, Peng J, Pang J, Ma N et al (2020) RNA-Seq Reveals Underlying Transcriptomic Mechanisms of Bone Marrow-Derived Mesenchymal Stem Cells in the Regulation of MicrogliaMediated Neuroinflammation After Subarachnoid Hemorrhage. Stem Cells Dev 29(9):562-573. doi:10.1089/scd.2019.0216

28. Zhang Y, Chopp M, Meng Y, Katakowski M, Xin H, Mahmood A et al (2015) Effect of exosomes derived from multipluripotent mesenchymal stromal cells on functional recovery and neurovascular plasticity in rats after traumatic brain injury. J Neurosurg 122(4):856-867. doi:10.3171/2014.11.jns14770

29. Zhang Y, Chopp M, Zhang ZG, Katakowski M, Xin H, Qu C et al (2017) Systemic administration of cell-free exosomes generated by human bone marrow derived mesenchymal stem cells cultured under $2 \mathrm{D}$ and $3 \mathrm{D}$ conditions improves functional recovery in rats after traumatic brain injury. Neurochem Int 111:69-81. doi:10.1016/j.neuint.2016.08.003

30. Zhao C, Wu H, Qimuge N, Pang W, Li X, Chu G et al (2018) MAT2A promotes porcine adipogenesis by mediating H3K27me3 at Wnt10b locus and repressing Wnt/ $\beta$-catenin signaling. Biochim Biophys Acta Mol Cell Biol Lipids 1863(2):132-142. doi:10.1016/j.bbalip.2017.11.001

\section{Figures}

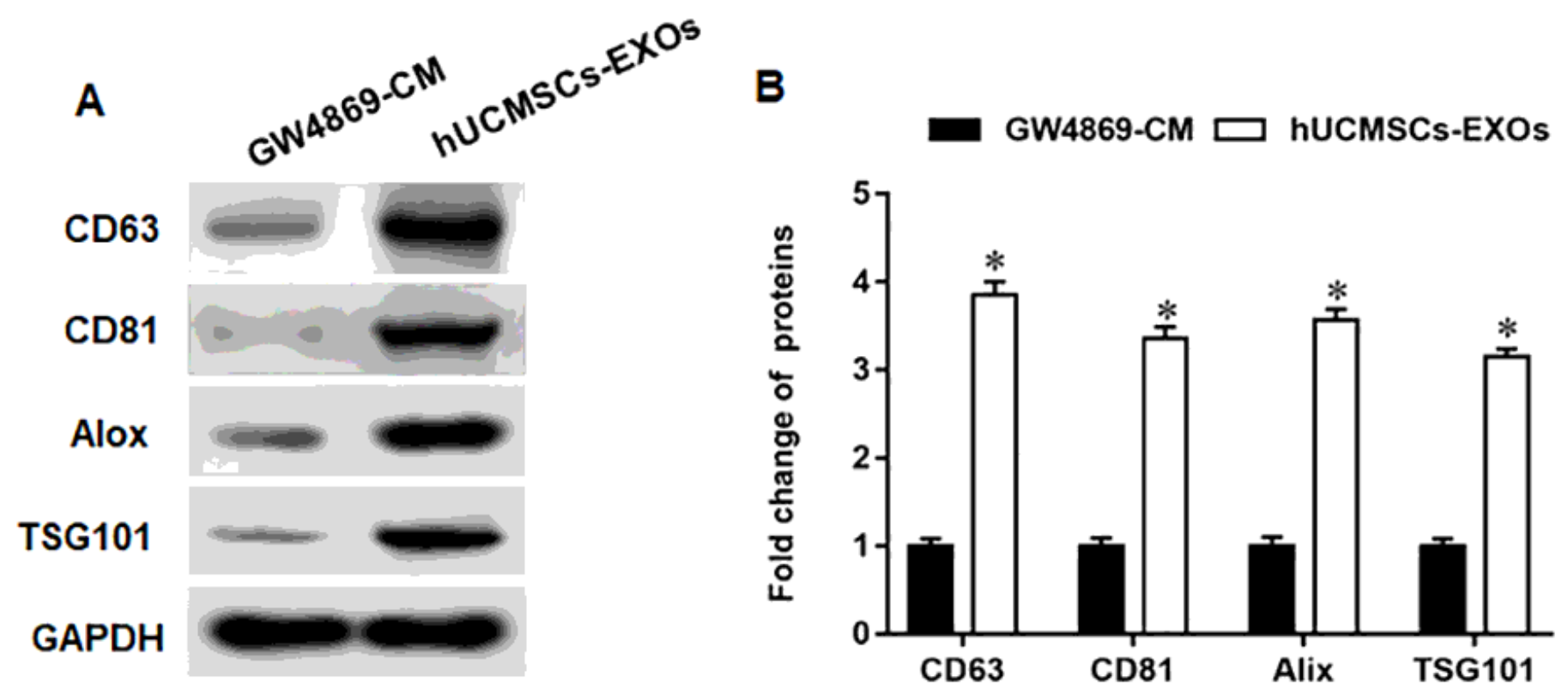

Figure 1 
Identification of exosomes derived from hUC-MSCs Cell culture supernatant was collected and exosomes derived from hUC-MSCs were extracted by exosomes extraction kits. The relative expression of exosomes marker proteins including CD63, CD81, TSG101 and Alix was detected by Western botting. Conditioned medium supplemented with exosomes inhibitor GW4869 was used as control. $\beta$-actin was used as an internal reference. $\mathrm{N}=6,{ }^{*} \mathrm{P}<0.01$.

A

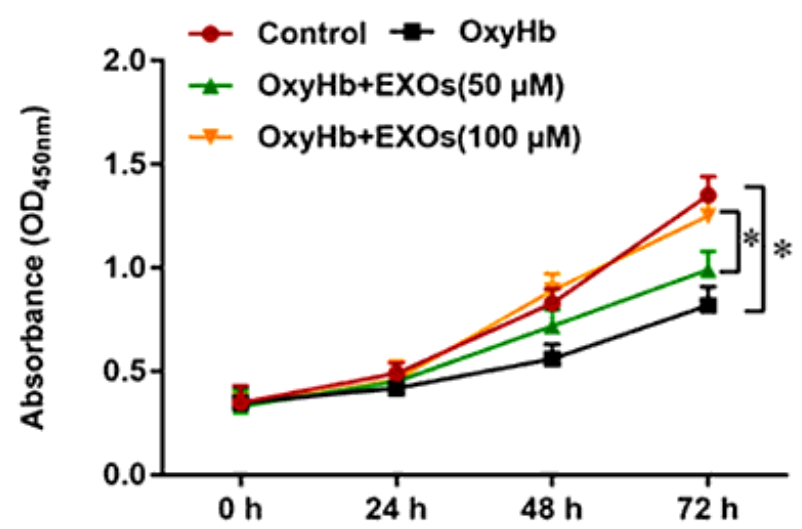

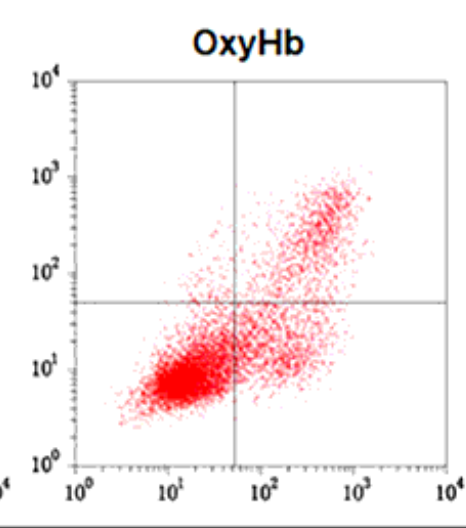

B
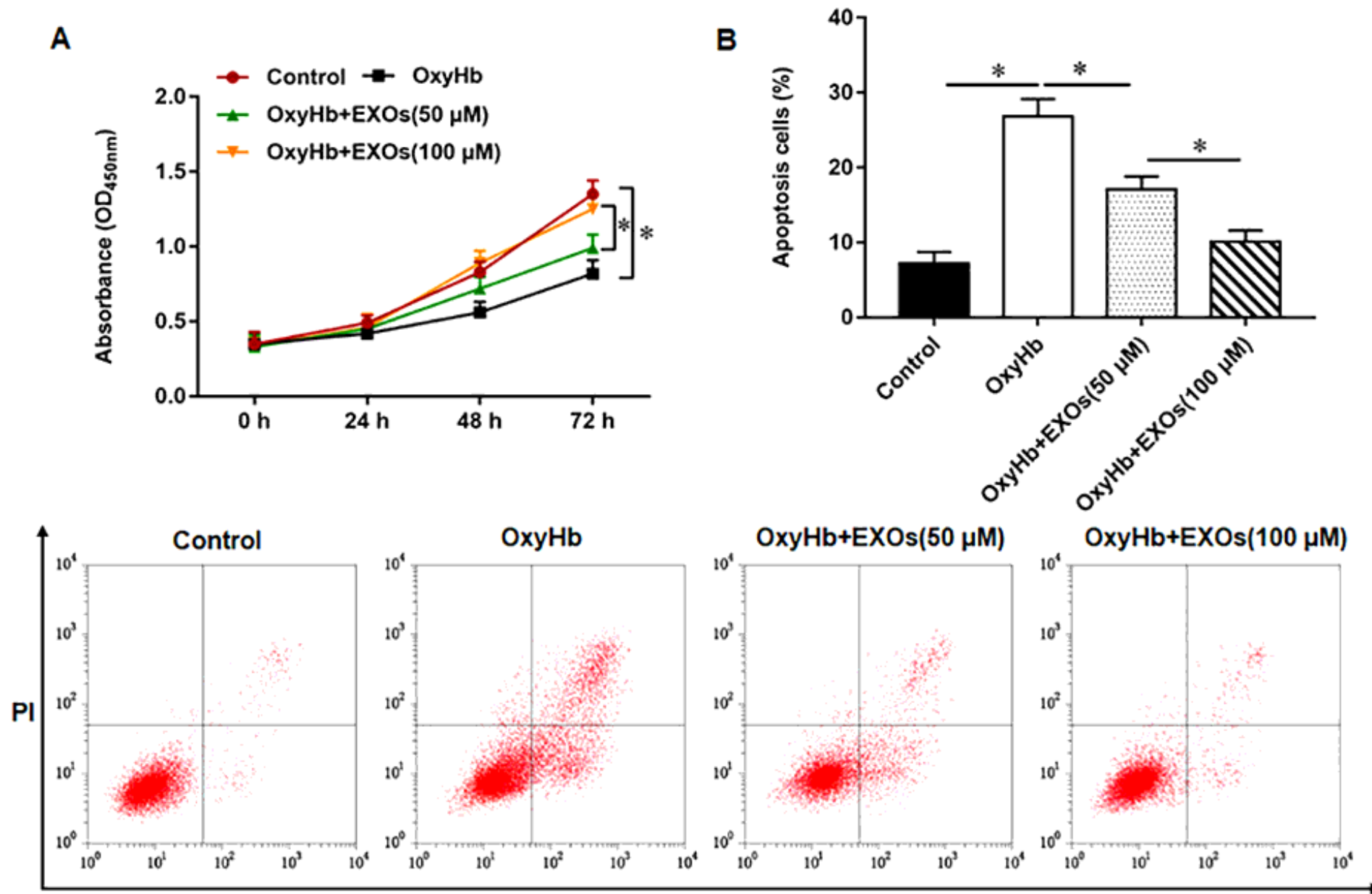

Annexin VFITC

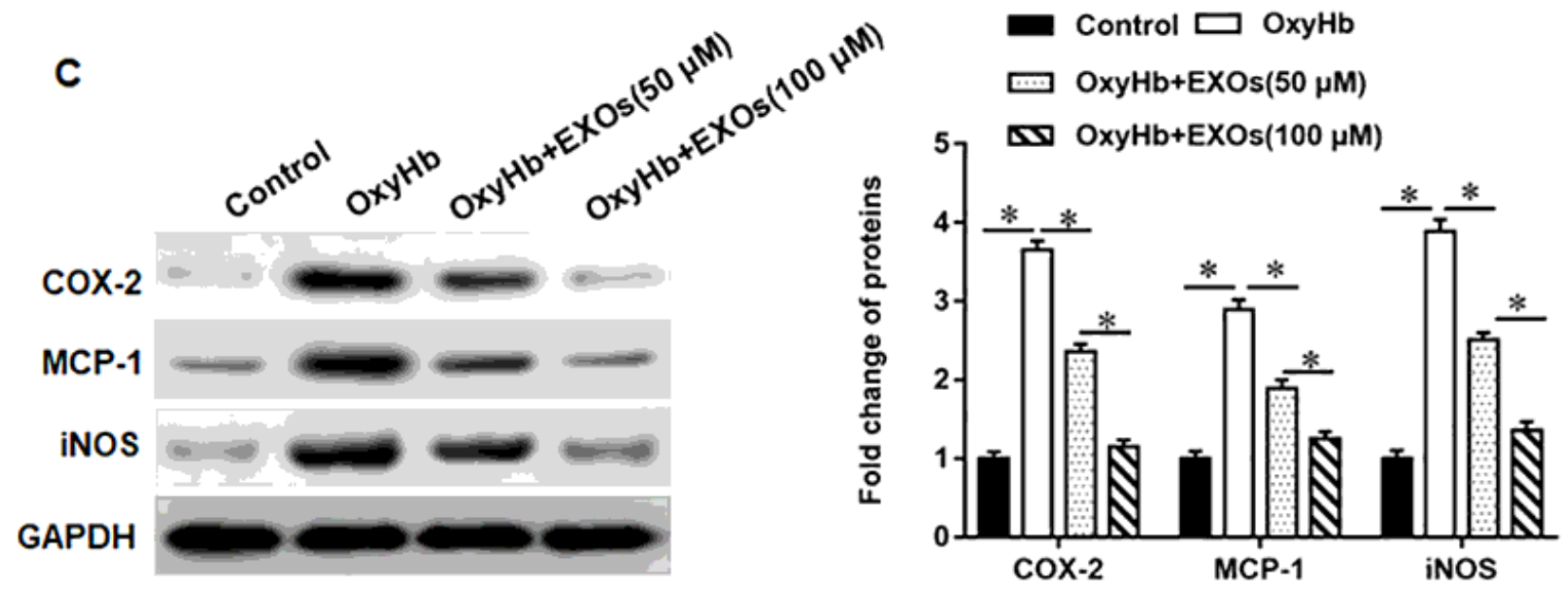

Figure 2 
Effects of exosomes derived from hUC-MSCs on oxyHb-induced PC12 cells PC12 cells were stimulated with $50 \mathrm{ng} / \mathrm{mL}$ oxyHb (cell density was $1 \times 106$ ) for $6 \mathrm{~h}$, and then cells were then incubated with exosomes at concentrations of 50 and $100 \mu \mathrm{g} / \mathrm{mL}$, respectively. A. MTT assay was used to detected cell proliferation. B. Apoptosis of PC12 cells were detected by flow cytometry. C. The protein expression of COX-2, MCP-1 and iNOS was analyzed by Western botting. $\beta$-actin was used as an invariant internal control for calculating protein-fold changes. $N=6,{ }^{*} P<0.01$.
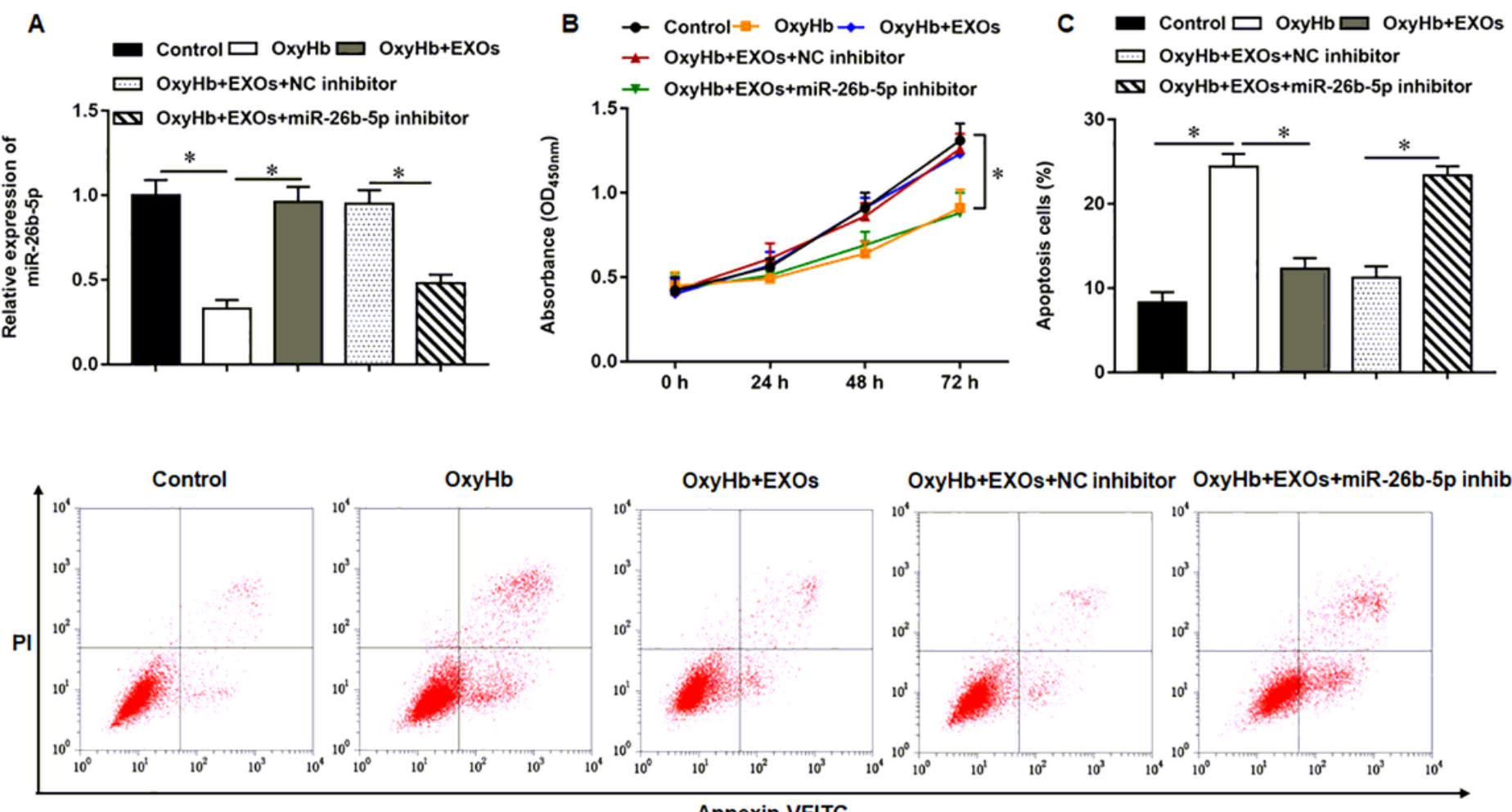

Annexin VFITC

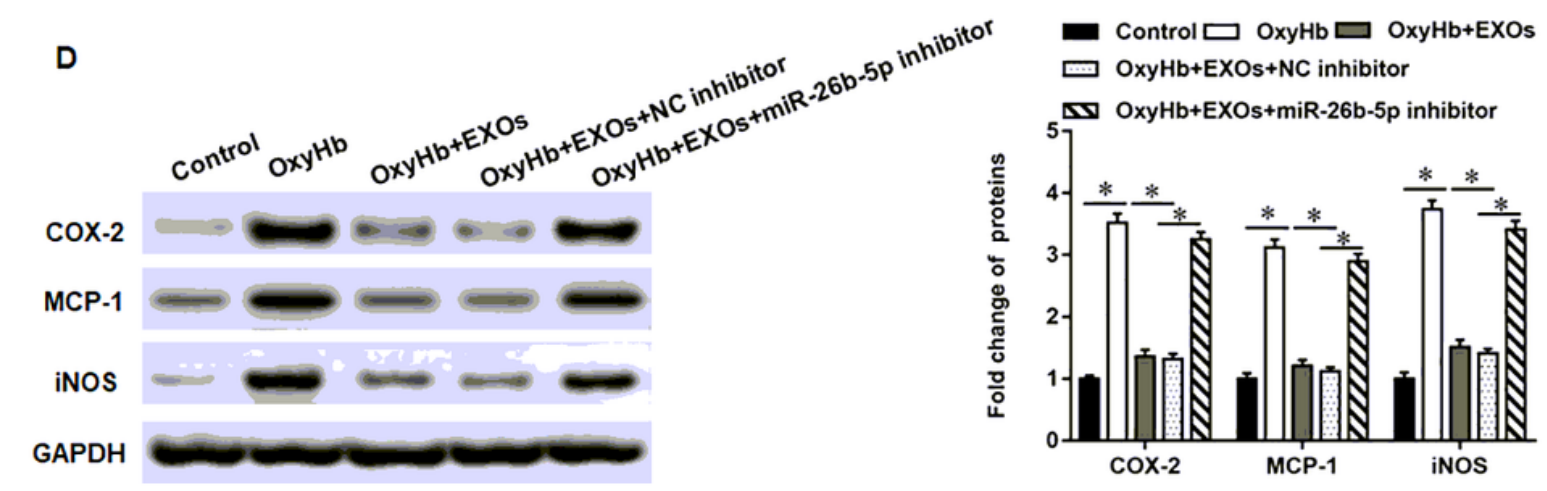

\section{Figure 3}

Effects of exosomes derived from hUC-MSCs modified by miR-26b-5p on oxyHb-induced PC12 cells PC12 cells were stimulated with $50 \mathrm{ng} / \mathrm{mL}$ oxyHb (cell density was $1 \times 106$ ) for $6 \mathrm{~h}$ The cells were then incubated with exosomes at a concentration of $100 \mu \mathrm{g} / \mathrm{mL}$ and transfected with miR-26b-5p inhibitor. A. Relative expression of miR-26b-5p was detected by RT-qPCR. B. MTT assay was used to detected cell proliferation. C. Apoptosis of PC12 cells were detected by flow cytometry. D. Western botting was used to analyzed the protein expression of COX-2, MCP-1 and iNOS. $\beta$-actin was used as the loading control. $\mathrm{N}=6$, ${ }^{*} \mathrm{P}<0.01$. 
A

MAT2A-wt : 5' ggaaAUCCCUUCA-UACUUGAa 3'

$\|||||$

miR-26b-5p : 3' uggaUA-GGACUUAAUGAACUu 5'

MAT2A-mut : 5' ggaaAUCCCUUCA-UACUUGAa 3'

B
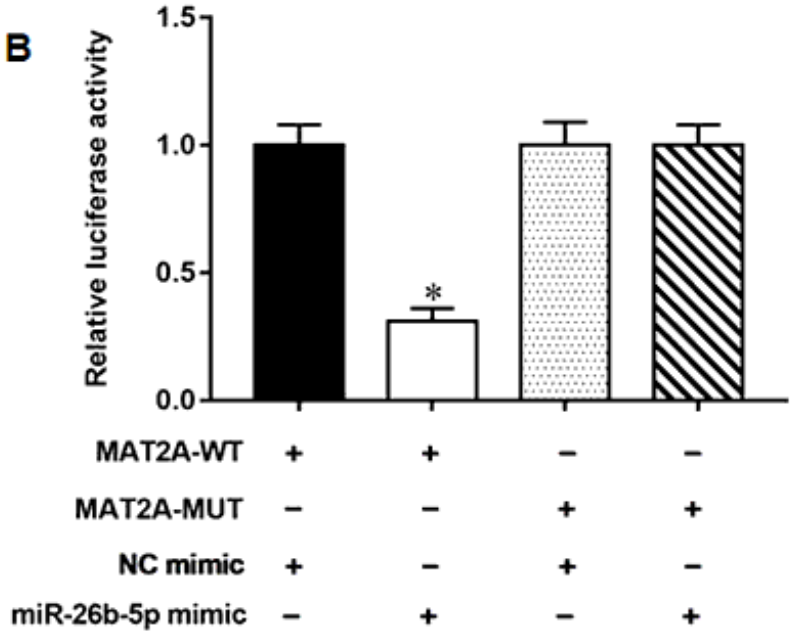

C

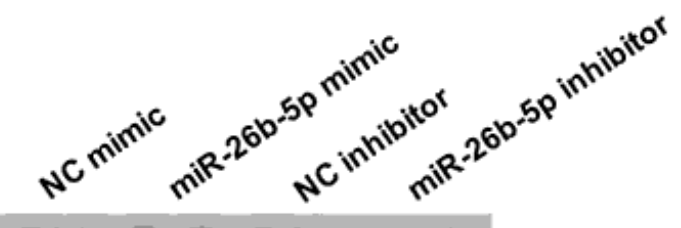

MAT2A

GAPDH

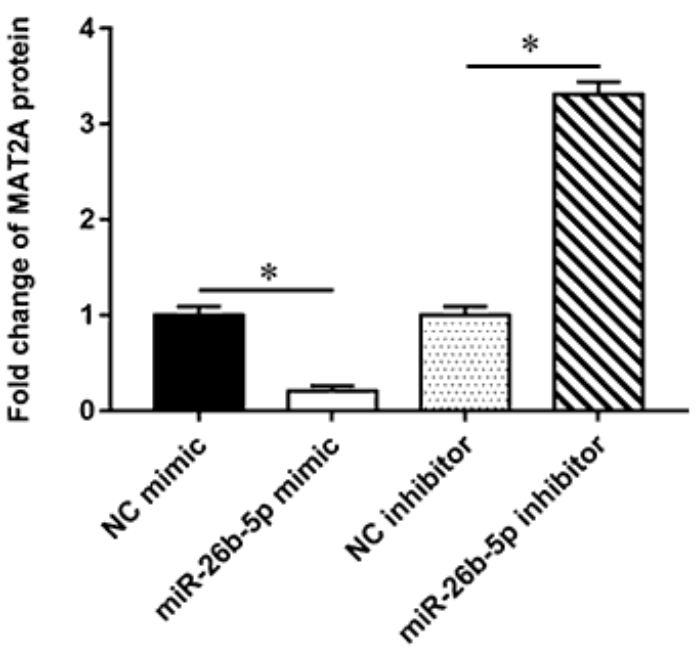

\section{Figure 4}

MAT2A was a direct target of miR-26b-5p A. StarBase3.0 (http://starbase.sysu.edu.cn/) was used to predicted the targeting sites of miR-26b-5p and MAT2A. The wild-type and mutant sequences of MAT2A are shown in the figure. $B$. The relative luciferase activity was tested with wild-type and mutant-type of MAT2A, respectively. C. The miR-26b-5p mimic or miR-26b-5p inhibitor was transfected into cells and the protein expression of MAT2A was analyzed by Western blotting. $\beta$-actin was used as an internal reference. $N=6,{ }^{*} P<0.01$. 


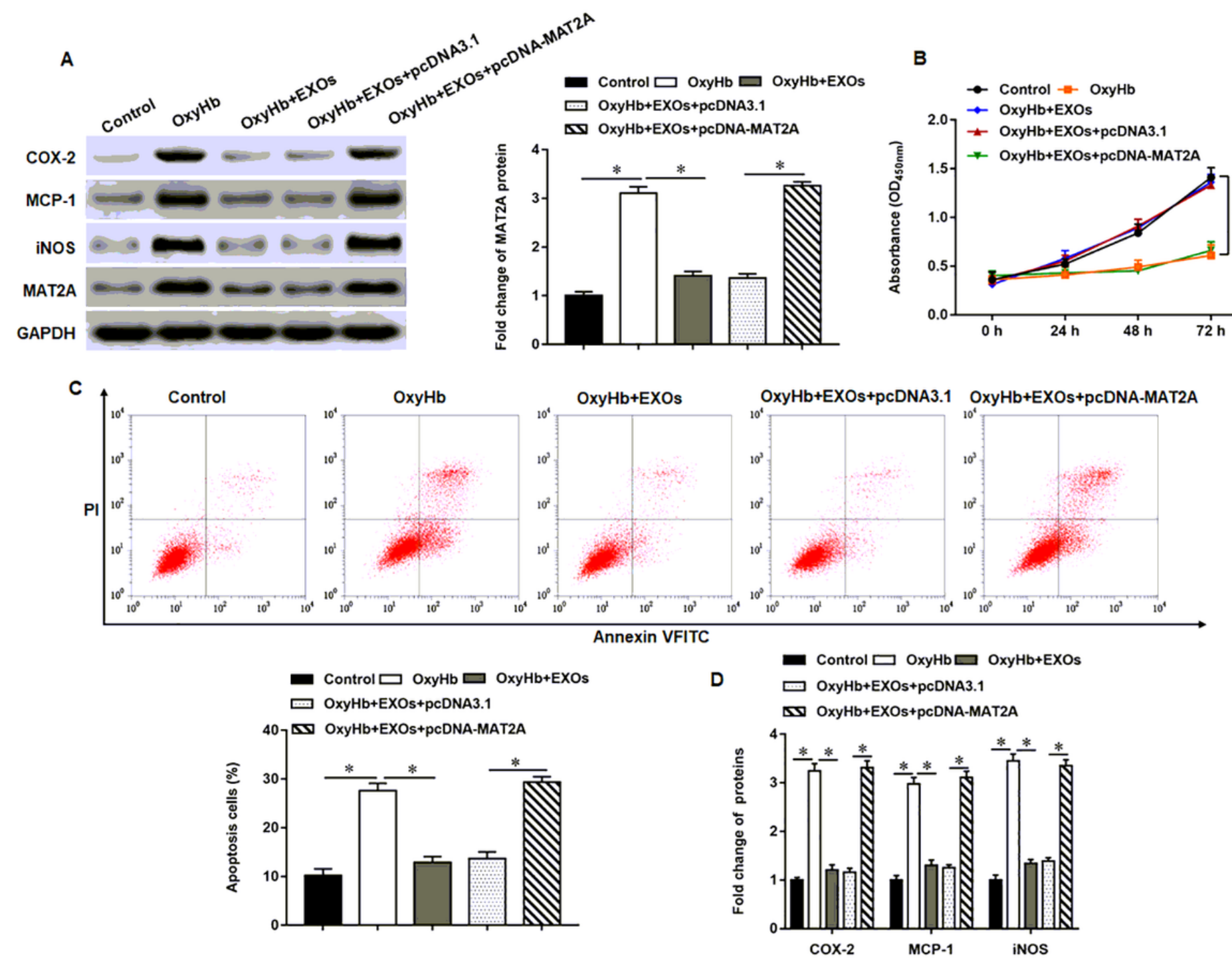

Figure 5

Effect of regulating MAT2A expression on oxyHb-induced PC12 cells PC12 cells were stimulated with 50 $\mathrm{ng} / \mathrm{mL}$ oxyHb (cell density was $1 \times 106)$ for $6 \mathrm{~h}$. The cells were then incubated with exosomes at a concentration of $100 \mu \mathrm{g} / \mathrm{mL}$, and the overexpression vector of MAT2A was transfected into the cells. A. The protein expression of MAT2A was analyzed by Western blotting. B. MTT assay was used to detected cell proliferation. C. Apoptosis of PC12 cells were detected by flow cytometry. D. Western botting was used to detected the protein expression of COX-2, MCP- 1 and iNOS. $\beta$-actin was used as an invariant internal control for calculating protein-fold changes. $N=6,{ }^{*} P<0.01$. 


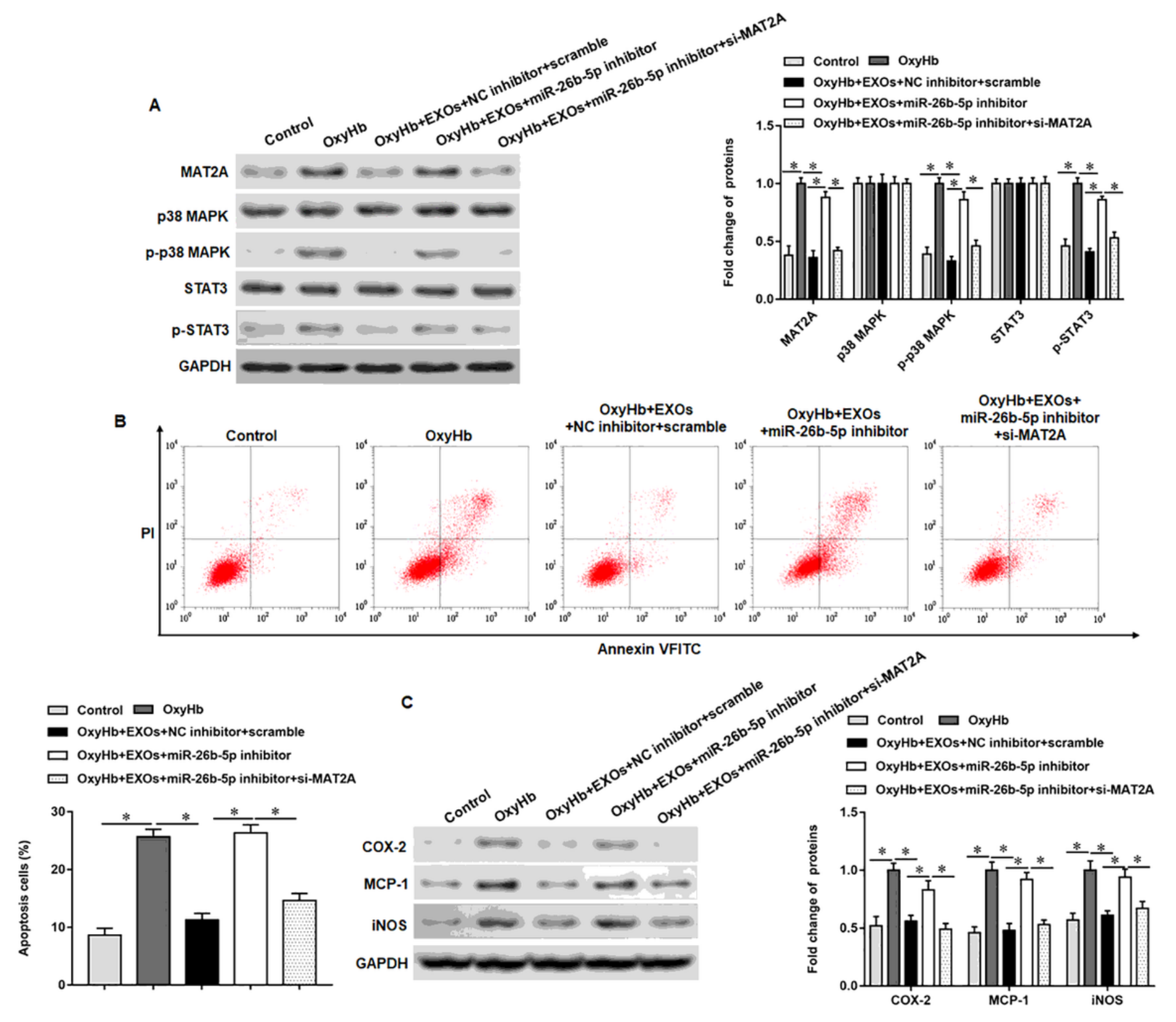

Figure 6

MiR-26b-5p-modified exosomes inhibited p38 MAPK/STAT3 signaling pathway to alleviate oxyHbinduced cell injury PC12 cells were stimulated with $50 \mathrm{ng} / \mathrm{mL}$ oxyHb (cell density was $1 \times 106$ ) for $6 \mathrm{~h}$ and incubated with exosomes at a concentration of $100 \mu \mathrm{g} / \mathrm{mL}$. Then, cells were transfected with miR-26b-5p inhibitor alone or together with si-MAT2A, respectively. A. Western botting was used to detect the protein expression of MAT2A as well as the phosphorylation levels of p38 MAPK and STAT3 proteins. B. MTT assay was used to detected cell proliferation. C. The protein expression of COX-2, MCP-1 and iNOS was analyzed by Western botting. $\beta$-actin was used as an internal reference. $N=6, * P<0.01$. 


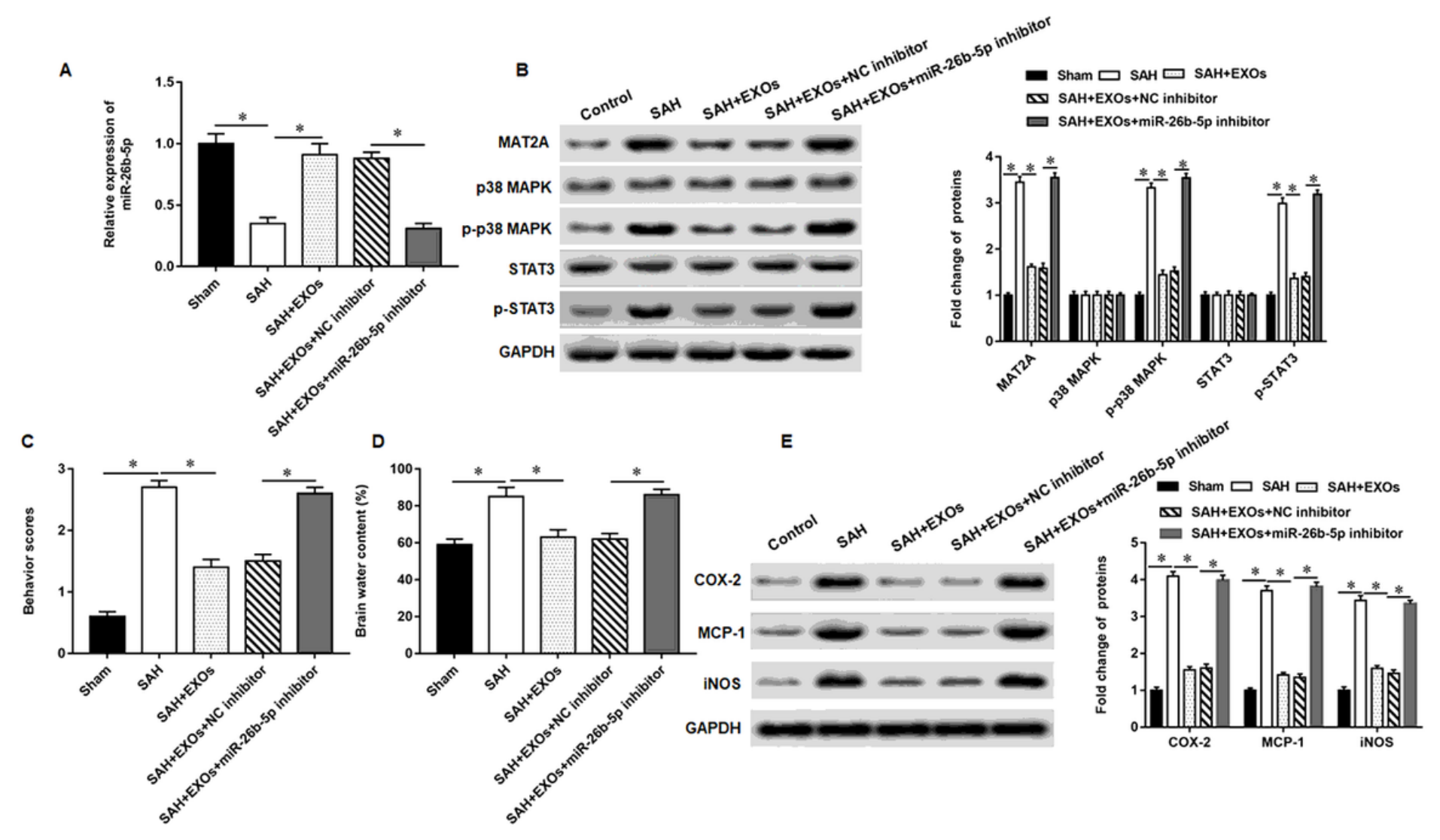

Figure 7

MiR-26b-5p-modified exosomes attenuate early brain injury in SAH rats The animal model of SAH was established by the classical occipital cistern secondary injection method. Thirty SD rats were divided into five groups on average. The cerebellar medullary cistern of rats in sham operation group was injected with normal saline. A. Relative expression of miR-26b-5p was detected by RT-qPCR. B. Western botting was used to detect the protein expression of MAT2A as well as the phosphorylation levels of p38 MAPK and STAT3 proteins. C. Behavioral and neurological functions of SAH rats were evaluated $24 \mathrm{~h}$ after modeling. D. Brain edema content in SAH rats was detected $24 \mathrm{~h}$ after modeling. $\mathrm{E}$. The protein expression of COX-2, MCP-1 and iNOS was detected by Western botting. $\beta$-actin was used as an invariant internal control for calculating protein-fold changes. $N=6,{ }^{*} P<0.01$. 